\title{
EMPYEMA THORACIS
}

\author{
By Kenneth S. MullaRd, F.R.C.S.
}

Thoracic Surgeon, Harefield Hospital

Empyema has largely changed its character of recent years. While formerly the problem was that of the treatment of an acutely ill and toxic patient, now, with effective chemotherapy, it has become a technical matter of removing a collection of fluid, with the least possible risk and with the speediest possible return to full recovery, from a patient who is seldom in any immediate danger. Since the surgical treatment of empyema ceased to be urgent and life-saving, more attention has been paid to the avoidance of chronicity, and to insistence on full functional recovery, matters which received scant attention in the past. This article is concerned, therefore, chiefly with the detailed management of acute empyema and reviews briefly the aetiology, pathology and diagnosis of empyema, together with chronic and tuberculous empyema.

\section{Aetiology}

Most empyemata arise as a complication of infection in the underlying lung. This infection is usually a primary pneumonia due to a specific organism, the pneumococcus, streptococcus, staphylococcus, Friedlander's bacillus, etc. The purulent effusion in the pleural cavity may collect while the lung infection persists, or it may first become apparent when the lung infection is subsiding. This is as true of pneumonia treated by chemotherapy as it was of those not so treated, but the division into syn-pneumonic and postpneumonic empyema is of little practical value today. Besides primary pneumonia, infection in the lung may be due to inhaled material from dental, sinus or pharyngeal infection; inhaled septic material is far more likely to cause pneumonia and empyema than the classical obstructive lung abscess. Lung infection may be secondary to an inhaled foreign body or, most important; secondary to a carcinoma of bronchus. Empyema in association with carcinoma of bronchus is usually a late phenomenon, found at a stage when there is extensive infection and destruction of lung distal to a bronchus obstructed by a growth which has, by this time, spread widely in the mediastinum and elsewhere. This picture is so often seen that empyema is sometimes regarded as contraindicating pneumonectomy for carcinoma; but an empyema may occur at an early stage, when resection is still practicable and worth whilew (Fig. I). Such an empyema is a token of thevirulence of the infection trapped beyond the bronchial obstruction, and is not, in itself, evidence $\vec{\omega}$ that the growth itself is far advanced. Empyemas may be provoked by an infected pulmonaryo infarct; it may sometimes occur in actinomycosis 3 and it may be secondary to a flare up of infection in a bronchiectatic lung.

So much for empyema secondary to lungor infection. The next main group consists of empyema secondary to direct injury or infection ${ }_{0}^{+}$ of the pleura. Penetrating or non-penetrating wounds and injuries may be responsible. Infected haemothorax is the usual precursor of franko empyema in this group. Empyema occurring as? a complication of thoracic operations falls intothis group. After most intra-thoracic operatiogs there is a pleural effusion, provoked partly mechanical irritation of the pleura and partly the irritation of blood oozing from the operative site. Rapid re-expansion of the lung to obliterateo the dead space, together with removal of theू effusion by aspiration or drainage, and adequate 2 chemotherapeutic ' cover' are the best safeguards $\overrightarrow{0}$ against the development of empyema.

Traumatic rupture of a bronchus is a cause of empyema which is often overlooked. A crush. injury of the chest may, in addition to causing aO haemothorax, shear across a bronchus. The resulting empyema is, of course, quite intractable unless the causative lesion is recognized. The empyema is due to the infection spreading from? the lung, from which secretions cannot drain effectively. Direct contamination of the pleurab space from the torn bronchus does not occur? since there is not complete disruption of alE. components of the bronchial wall ; the effect of the injury to the bronchus is to cause a bronchia stenosis. Direct contamination of the pleurap space from the bronchus does occur, however as a complication of all forms of pulmonary re? section should the suture of the bronchial stumf break down.

Empyema may be due to injury to the oeso $\stackrel{?}{?}$ phagus. When the mucosa alone is damaged mediastinitis follows, but if gross rupture of ald layers, including mediastinal pleura, occurs, as in 

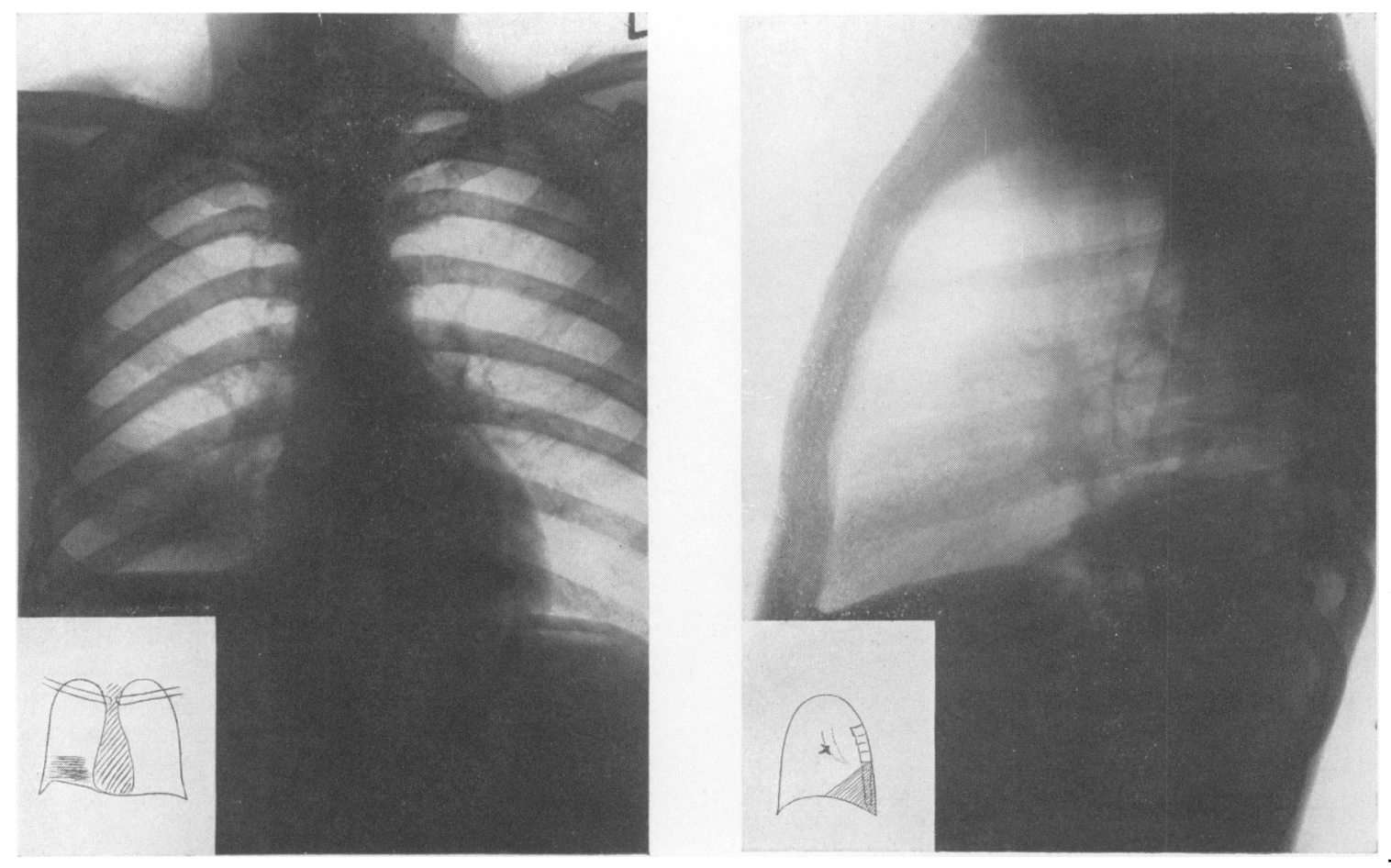

FIG. I.-An empyema following an acute pulmonary infection in a man of 34 , with no previous history of chest disease. The empyema was due to a squamous-cell carcinoma of the right lower lobe bronchus.

most cases of spontaneous rupture, and sometimes following instrumentation, then empyema or pyo-pneumothorax results.

A further group comprises empyema due to causes other than pulmonary infection or direct infection of the pleura. A sub-phrenic abscess will usually provoke a pleural effusion above it, which is at first sterile. If the sub-phrenic abscess remains untreated an empyema may develop, either from rupture of the abscess into the pleural space or by lymphatic spread. Liver abscess follows a more chronic course and if it ruptures through the diaphragm it does so at a late stage, when the pleural space has been obliterated by adhesions, and the abscess discharges into the lung.

Empyema thoracis has been defined as a collection of pus in the pleural space. It is well to remember that collections of pus may occur in the extra-pleural tissues. Breaking down mediastinal glands, or lower deep cervical glands, may cause an extra pleural ' empyema ' (Fig. 2). Osteomyelitis of a rib may do likewise (Fig. 3).

The final group consists of tuberculous empyema. Most of these cases occur as a complication of artificial pne.umothorax therapy. The spontaneous rupture of adhesions, the rupture of a cavity wall, and too extensive adhesion section in which tuberculous foci are opened are obvious causes, but the great majority arise insidiously during the course of treatment by artificial pneumothorax of pulmonary lesions in which atelectasis of a segment, lobe or lung is a feature. The cause of the empyema in these cases is uncertain. Empyema is also likely to occur when a pneumothorax is given up after some years of treatment, an effusion developing which readily acquires all the characteristics of a thick tuberculous empyema. Contamination by faulty aspiration, or rupture of a pure tuberculous empyema into the lung with the production of a broncho-pleural fistula, or ill-advised drainage, are the usual caluses of a tuberculous empyema becoming a mixed infection empyema.

\section{Pathology}

The striking feature of the microscopic pathology of non-tuberculous empyema is that the pleura appears normal. To one side is lung tissue, which may show evidence of the infection which gave rise to the empyema. To the other side is 


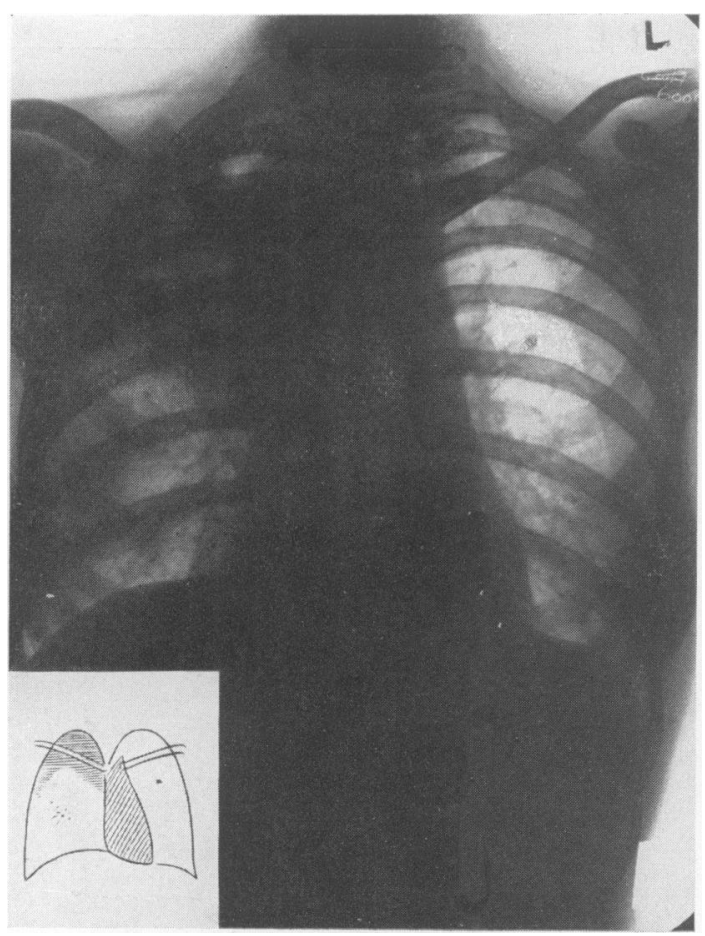

FIG. 2.-An extra-pleural 'empyema' in a girl of 25. Breaking down tuberculous glands in the mediastinum and lower deep cervical group caused a cold abscess, which pointed in the supra-clavicular triangle. Secondary infection developed, but the abscess never ruptured into the pleural space or into the lung.

the layer of fibrin and pus cells. To the naked eye, the fibrin layer may be thin and filmy, or half an inch in thickness and of the consistency of cardboard or cartilage. The sharp angles which are found in the normal pleural space, such as the costo-phrenic angle and the angle between the aorta and paravertebral gutter, are filled with fibrin and obliterated, so that on opening an empyema widely it is difficult to make out landmarks. The fibrin layer passes smoothly from the lung to the diaphragm and chest wall. At the margins of the empyema there is an abrupt change from the fibrin-covered pleura to normal pleura. Unless an interlobar empyema is present, the margins of the fissures will be sealed by the fibrin layer passing over them, but when this layer is divided, the underlying fissure shows light adhesions only. Similarly, the fringe of the lower lobe is densely adherent to the diaphragm, but the main diaphragmatic surface of the lobe is little involved. The mediastinal surface of the lung is seldom involved, since an empyema usually arises parietally, and the lung becomes lightly adherent to the mediastinum before a dense fibrin lay can form on it.

This description almost holds true for tubes culous empyema, except that a thick fibrin layer is commonly found. The important difference between the two is that in tuberculous empyenfa the underlying lung frequently shows fibro: septa running at right angles to the pleura in the parenchyma. Whether this feature is th result of the empyema, or of the pulmonary tubee culosis, is difficult to say. More probably it is part of a fibrotic process following long-standing collapse of the lung, as it is sometimes seen in chronic non-specific empyema. The viscerat pleura is intact, as in non-specific empyema, big areas can be found where the tuberculous proces in the lung has reached the surface and destroyed it. The contiguous parietal pleura may also be involved, so that there is no trace of the pleurat membranes in these areas, all being replaced by fibrous tissue or tuberculous granulation tissue These two points in the pathology are responsibfe for the difficulty in obtaining re-expansion of the lung following decortication, in those few cases im which re-expansion is desirable.

\section{Diagnosis}

Diagnosis in the first place depends uponagke clinical finding of the signs of fluid in the plemiral space. The next step is to obtain posteres anterior and lateral radiographs to confirm the situation and extent of the effusion. The dia $\overline{6}$ nosis is made absolute by aspiration of some of the fluid, part of which is sent for cytological examina tion and culture, part being retained for con $\overrightarrow{-}$ parison with later specimens. Having the knowledge that an empyema is present, and with the radiographs at hand, the case should be reviewed and the exact cause of the empyema determines. An insidious onset will give rise to suspicion of an underlying carcinoma, but a sudden and apparent typical pneumococcal pneumonia preceding the empyema by no means rules out carcinoma. Careful questioning as to any preceding change of habitual cough, chest pain or vague ill-health th the months before the acute episode should never be omitted. Inspection of the radiographs mat suggest enlargement of the lymph glands at tore hilum, or widening of the superior mediastinum. The mediastinum may not be shifted to the side opposite the empyema, or may be drawn towargs the side of the empyema, suggesting atelectasts of the lung beneath the effusion. If there is any suggestion, from these points, that a carcinoma may be present, bronchoscopy should be carried out. This will reveal a carcinoma, if present, about three-quarters of the cases. In the remaigder, the growth is situated peripherally in the 

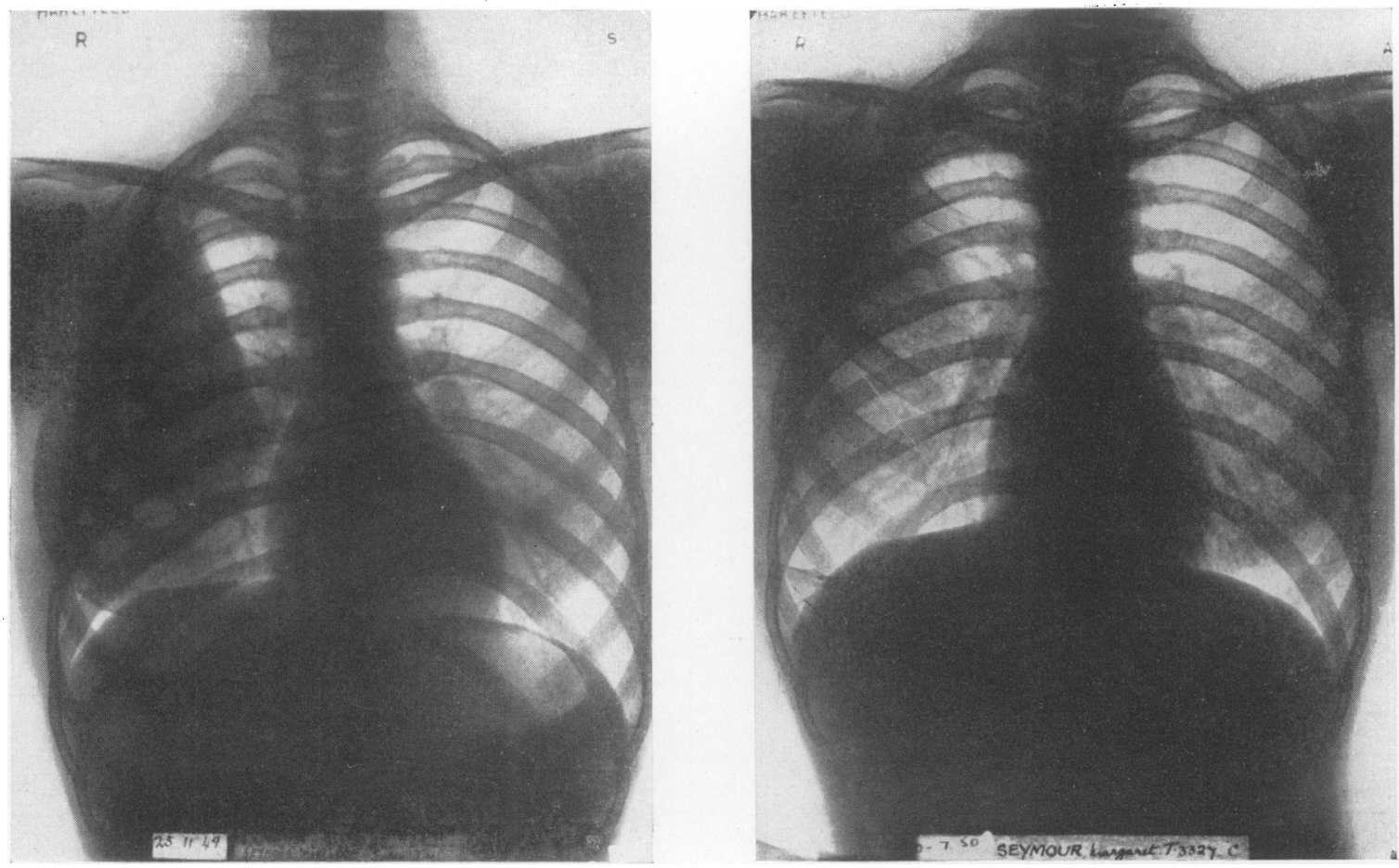

Fig. 3.-An extra-pleural 'empyema' in a girl of 13 , secondary to staphylococcal osteomyelitis of the third rib. The sequestration of the rib, and the periosteal reaction is obscured by the density of the pus in the normal film (a), but can be seen in the penetrating film (b).

lung, beyond the range of the bronchoscope. In these cases, a penetrating film may show a mass, or a film can be taken after as much fluid as possible has been aspirated. Culture of empyema pus is not often helpful in these days of chemotherapy, as it is usually sterile.

A history of pain and a troublesome, querulous patient complaining persistently of pain for which there appears insufficient reason, will, if ignored, cause difficulty in cases subsequently shown by pleural biopsy to be suffering from malignant endothelioma of the pleura (Fig. 4).

Radiographic evidence of collapse, with a history of chronic productive cough, suggests bronchiectasis, and it can be anticipated that treatment will be difficult.

The diagnosis has to be made from lung abscess. This is usually easily done on the history, signs and radiographic appearances. But a localized empyema may simulate a peripheral lung abscess, and vice versa, in their radiographic appearances. This difficulty is most likely to arise in the differentiation between a localized empyema in the paravertebral gutter and a lung abscess in the dorsal segment of the lower lobe. In the last resort, the diagnosis depends on the presence or absence of lung sloughs in the cavity. The presence or absence of profuse purulent sputum is no certain guide, since a bronchopleural fistula may develop from an empyema, and the sputum be as profuse and foul as that from a lung abscess.

It may be necessary to distinguish between a sub-phrenic abscess and a basal empyema. The two may co-exist. Drainage of the empyema alone will not relieve the condition. A most satisfactory method of determining the presence of a sub-phrenic abscess and its exact situation is to induce a pneumo-peritoneum, with 500 to $1,000 \mathrm{cc}$. of air, and to take radiographs in the postero-anterior and lateral planes, with the patient in the upright position. Air will pass up under the diaphragm and will separate the liver and diaphragm, anterior and posterior to the abscess, which will show as an area of adhesion between them. If no abscess is present, air will fill the entire sub-phrenic space, the liver will fall away, and the diaphragm, with the empyema above it, will be clearly outlined. There is no danger that this procedure will rupture a subphrenic abscess into the general peritoneal cavity 


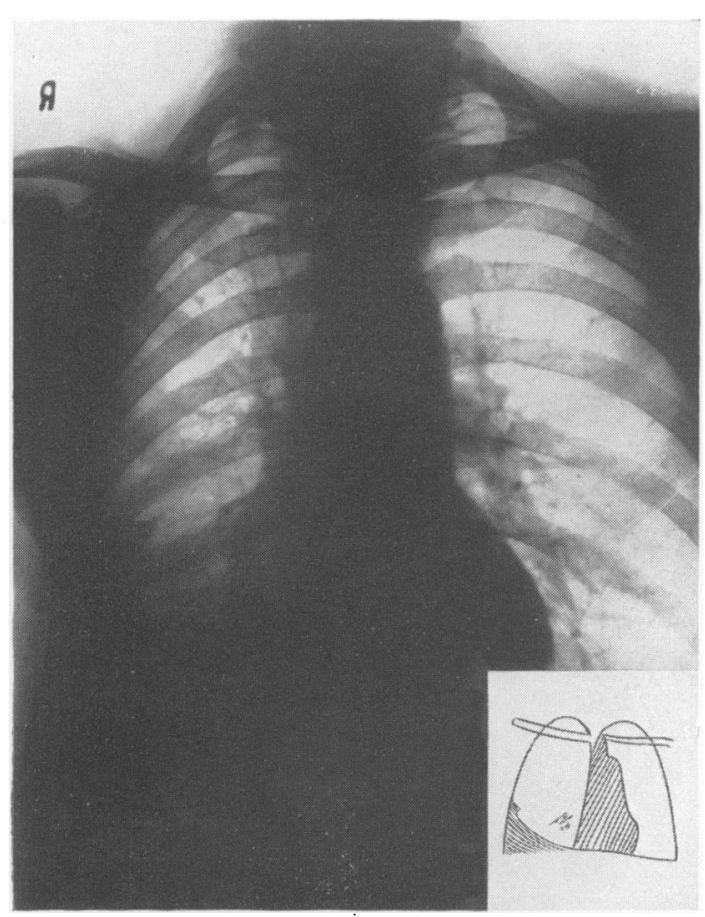

FIG. 4.-A small basal empyema from which sterile pus was aspirated, in a man of 50 . Persistent pain led to a diagnosis of pleural endothelioma, confirmed by pleural biopsy.

if it is carried out at the stage at which an empyema has had time to develop, i.e. some weeks after the peritoneal infection first occurred.

It remains to consider the differential diagnosis from tuberculous pleural effusions and empyemata. Nowadays most empyemata are found to be sterile, thanks to chemotherapy, but pus cells will be found, in varying quantity, depending on the type and age of the empyema. .A clear effusion, containing a few lymphocytes and a few polymorphs, is suspicious of tuberculosis. The age of the patient, the Mantoux reaction, the results of gastric lavage and laryngeal swab cultures for tubercle bacilli, culture of the fluid. itself for tubercle bacilli, the radiological appearances in the lung after aspiration of the fluid, the tendency or otherwise to reaccumulation of the fluid after aspiration, and the general progress of the patient will, between them, make the diagnosis, but this will only be done after some weeks in atypical cases. In the diagnosis of the clear sterile effusion, it must be remembered that a sudden atelectasis of a lobe, or even a segment, will usually provoke a small sterile effusion no matter what the cause of the atelectasis.

\section{Treatment}

In the introductory paragraph it was pointed ooft that the danger to life in the acute stage of entpyema has largely been removed by chemo:therapy. The good general condition of the patient whose empyema has been sterilized leads to a reluctance to impose upon him the discomfout of tube drainage, and there is a tendency to pesssist with aspiration for too long. Many empyema can be treated by aspiration alone, but there ame many pitfalls. A normal temperature and pulse chart certainly shows that infection is controlled, but gives no guarantee that a small, untapped loculus will not flare up at a later date, some tinge after chemotherapy has ceased. These pockets ase very difficult to find with the exploring needie. They show their presence by rupturing into the lung, when their contents are coughed up causing some pneumonitis in the process. All is then quiet for a time after this spontaneous drainage until the pocket reforms and again ruptures into the lung, until eventually the affected lobe develops considerable bronchiectasis.

Radiological control of the progress of aspir摩 tion treatment is unsatisfactory, especially in the later stages, when it is impossible to differentiate between fibrinous thickening of the pleura and small empyema too thick to be aspirated.

By keeping specimens of the pus aspirate advised by Barrett (1950), the process of thickering can be watched, but it is always possible thata thin loculus is being successfully aspirated and the a loculus of thick pus, which no longer core municates with it, is being neglected. Strepte kinase-streptodornase may be of value preventing the undue deposition of fibrin, but does

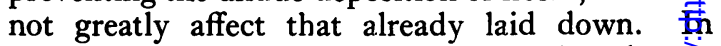
general, aspiration should not be continued onge the pus has become thick, or does not flow readíy through a needle. In practice this means thigt empyemata containing thin pus which can be removed entirely with a few aspirations spread over two or three weeks at most are suitable forr aspiration alone, but that for the remainder, sorge form of surgical treatment will be necessary. Aspiration is, further, of great value in infants aid small children who do not tolerate tube drainage readily.

Some technical points in aspiration of the chest are of importance. The patient must be corosfortably placed so that he will not tire and mo should the procedure be prolonged. The position must also be comfortable for the operator so that he can manipulate a syringe for a long time without moving the needle through fatigue. All aspirations should be carried out with a syringe with a two way tap. The joints between syringe, needle and tap must be of the locking type, not the ordinasy 


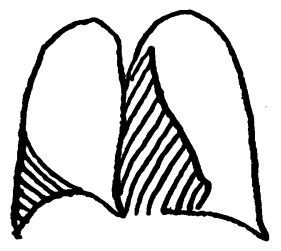

A
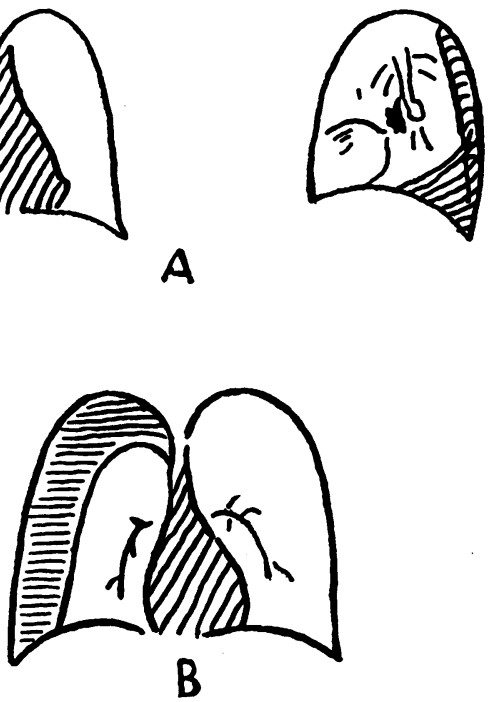

Fig. 5.-(a) A typical basal empyema which should heal within two or three weeks of open drainage. (b) A total empyema, which would probably take many weeks to close if treated by tube drainage; more rapid recovery can be achieved by decortication.

push-on fitting. The needle should be of wide bore. It is helpful to have an old artery forceps which can be clamped across the needle flush with the skin as soon as the needle point reaches the required depth and fluid appears in the syringe. The forceps prevents the needle being inadvertently introduced deeper, and should it be withdrawn a little during the aspiration the cause of the sudden cessation of flow of fluid can be seen at a glance, the forceps now lying away from the chest wall. It is of the greatest importance that no air should gain access to the pleural space, thus the insistence on the two-way tap with locking connections. If air is allowed to enter it will rise to the apex of the empyema cavity and will readily separate the pleural layers unless they are fortunately very firmly adherent. The pleural layers above the empyema being separated, a localized empyema is converted to a total pyopneumothorax. The simple act of disconnecting the syringe from the needle during aspiration may be the direct cause of prolonging the disability period from a matter of weeks to one of months or years.

If surgical treatment is required it is necessary to decide whether to employ open or closed drainage or decortication. Drainage is simple and safe; decortication (which is fundamentally an elaboration of closed drainage) involves a major thoracotomy, and must always carry an added risk.
Drainage, however, requires skilled and perhaps prolonged after-care, while decortication, once $z$ the immediate post-operative period is over, is $\stackrel{\mathbb{Q}}{\varrho}$ free of the problems of dressings and tube manage- $c$. ment. Experience shows that the typical basal $\overrightarrow{\vec{F}}$ empyema treated by drainage is healed in four weeks, and certainly within eight weeks. The patient, by the time the tube is removed, is $\frac{\bar{\sigma}}{\bar{\omega}}$ capable of full physical exertion and can return $\frac{\sigma}{\widehat{\sigma}}$ immediately to hard manual labour. Few patients $\propto$ will resume full work within eight weeks of de- ڤ) cortication, but should do so at about that time. $\overrightarrow{0}$ A total empyema, or an apical empyema, treated by drainage is likely to take longer than eight weeks $\vec{\omega}$ before the tube can be removed, and is therefore $\frac{\sigma}{D}$ suitable for consideration for decortication in order $\frac{0}{3}$ to reduce the disability period as much as possible $\frac{3}{i}$. (Fig. 5). Decortication is therefore to be considered where a long disability period is anticipated $\omega_{0}$ with simple drainage. Decortication is useful in very fat patients in whom tube management is $\vec{\phi}$ difficult. It is useful also when patients have to 0 travel long distances to obtain proper tube management. Rapid re-expansion of the lung following $\vec{z}$ either drainage or decortication, depends more

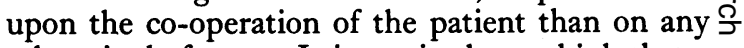
other single factor. It is a mistake to think that $\vec{\theta}$ patient who will not carry out exercises, etc., wi do any better with decortication.

Exercises, best supervised by a trained physige therapist, form an essential part of any treatment of empyema. The exercises are designed to main- $\bar{Q}$ tain an erect posture and to prevent the appearance $\frac{O}{\mathbb{Q}}$ of the earliest signs of the typical deformity of the $\varrho$ chronic empyema. Breathing exercises are de- $\overrightarrow{\overrightarrow{0}}$ signed to obtain full recovery of respiratory function. The exercises are localized expansion exercises against pressure, exerted by the physiotherapist's hand, or the patient's own hand, or by a belt. These localized exercises, in which attention is focused on each part of the chest in turn, 3 . apex, axilla, base and diaphragm, are of the greatest value in ensuring that the area of the chest $\frac{\circ}{3}$ affected by the empyema is not neglected and that 0 properly co-ordinated respiratory movements are restored. In addition, patients should engage in $\frac{D}{0}$ the most active pursuits possible, long walks, cycling, gardening, etc.

\section{Technique}

All empyemata should be drained in the sitting position. It is only in this position that drowning in a sudden flood of pus into the bronchial treec through a bronchopleural fistula can be avoided. Local or general anaesthesia can be used.

Closed intercostal drainage may rarely be necessary in an emergency and consists simply in intro- $\overrightarrow{\mathbb{Q}}$ ducing through the appropriate intercostal space $\frac{\mathbb{D}}{8}$ 

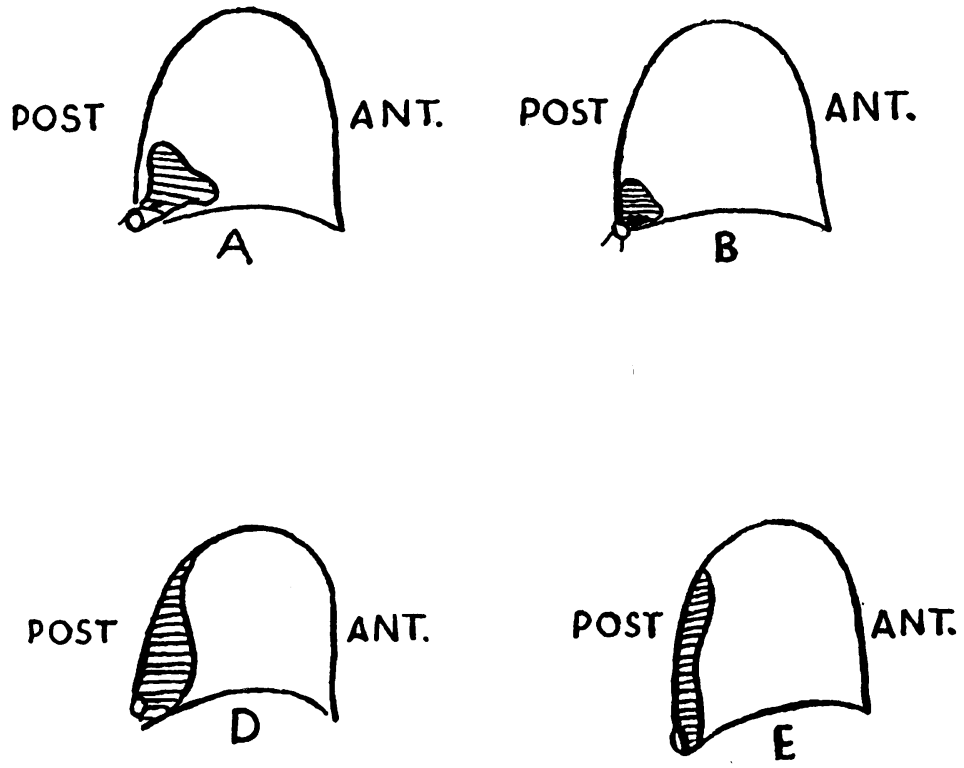
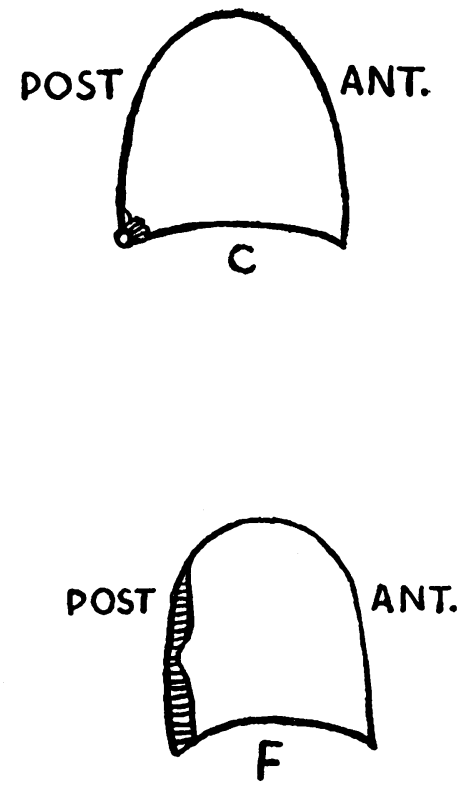

Fig. 6.-Progressive closing of empyema cavities, as seen on typical pleurograms. (a), (b) and (c), lateral views showing concentric shrinking of cavity towards the base; the tube is short and just enters the base of the cavity throughout. (d) and (e), paravertebral empyema. At the stage (e), it is necessary to lengthen the tube so that it reaches within an inch of the apex of the cavity if the stage (f) is to be avoided.

the largest trocar and cannula possible. The trocar is replaced by a tube connected to a water seal and the cannula withdrawn.

Almost always it is best to resect a rib to obtain adequate drainage. The situation of the most dependent point of the empyema cavity can be determined by instilling some lipiodol into the cavity and taking radiographs in the upright position. But whether this is done or not, a test aspiration should be carried out, with the patient in position for drainage, immediately prior to the incision. If no pus can be aspirated, it is wise to defer operation. The lowest point of the cavity being found, a vertical incision, 2 in. in length, is made over the rib selected. The muscle is divided, the periosteum incised and raised with an elevator. Two inches of rib are resected. The pleura is then opened sufficiently to allow a sucker to be introduced and the cavity evacuated. But the cavity cannot be sucked out in this way unless the walls of the empyema are firm and the mediastinum thus rendered stable. If these conditions do not obtain, opening of the pleura will cause a sucking pneumothorax and the patient's condition will deteriorate rapidly. All that can or should be done in these circumstances is to introduce a tube into the pleural cavity and to close the wound closely around the tube. This tube is connected to a water seal. This procedure of closed drainage has its prime indication in the conditions described above; that is, in the drainage of an empyema in which open drainage would result in an open pneumothorax.

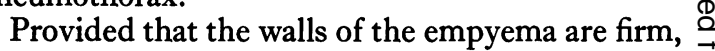
no respiratory disturbance will follow opening the pleura. The cavity is sucked out and the pleura then opened widely to allow fibrin clots to be removed. The cavity is gently explored and, if loculated, free drainage of the loculi ensured. $A$ 흑 routine biopsy of the pleura should now be taken. A straight wide-bore tube ( $\mathrm{I}$ in.) is then placed so that its inner end projects $\frac{1}{2}$ in. into the pleural cavity. Penicillin-sulphathiazole powder is then dusted into the wound, which is closed loosely around the tube. A safety pin is passed through the tube flush with the skin and secured with strap- $\frac{7}{0}$ ping. No dressings are placed between the skin and the pin. (If they are so placed, the tube is $N$ liable to be displaced when the dressings are $N$ changed, or a greater thickness of dressings will be N replaced, so that the tube will be withdrawn 0 somewhat. It is simplest and best to have no dressings beneath the pin.) No side holes are cut $\stackrel{\circ}{=}$ in the tube, either at its first insertion, or subse- $\stackrel{\mathcal{Q}}{\rightarrow}$ quently. Granulation tissue will grow into the side holes, so that pain and bleeding occur when the tube is removed for cleaning, and the button of granulation tissue will prevent the tube being 

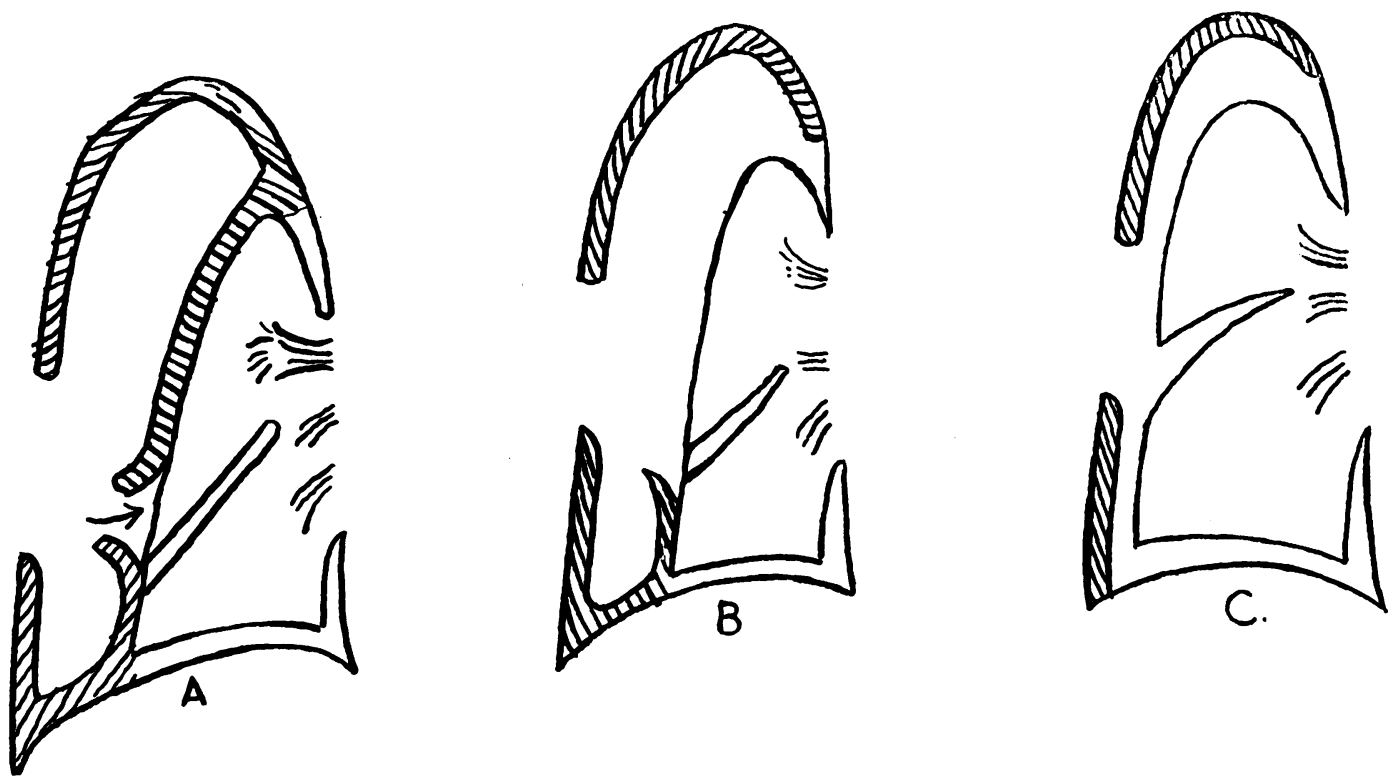

Fig. 7.-Decortication. (a) Beginning raising the fibrin layer from the visceral pleura. (b) The upper lobe freed. (c) Completed. The entire visceral pleura, including the fissures, has been freed, and the lung expands readily. The diaphragm has also been freed.

replaced to its correct depth. At the time of operation the tube can conveniently be left long, so that it can be attached to a water seal for a few days. This will allow the heavy discharge of pus during this period to be conveyed out of the bed away from the patient and will thus avoid frequent changes of dressing. The gentle suction of the water seal may help re-expansion of the lung, but in this case, where the empyema walls are firm, the water seal and closed drainage are a convenience and a help, as distinct from the case described above, of the prime indication for closed drainage, where it is a vital necessity.

After a few days, when the heavy discharge has settled, the tube is cut short, $\frac{1}{2}$ in. from the pin, and the patient gets up and begins to acquire the mobility and activity which is his best assurance of rapid re-expansion and healing.

The subsequent management of the case can be divided into two parts. First is the encouragement of correct breathing and posture, and secondly the detailed management of the tube. If the empyema has been correctly drained, the discharge will diminish to a very small amount of inoffensive pus. Provided this occurs the tube may be left in position for two to three weeks, when it is removed and the size and shape of the cavity determined by running lipiodol into the sinus. Films are taken in two planes. The tube is replaced as soon as the films have been taken since the sinus contracts very rapidly, and if the tube is left out for half ar hour it may be difficult to replace. The site of the skin sinus should be marked with a radio-opaque marker-a curtain ring serves-so that its position is apparent on the films. Unsatisfactory pleuro- $\frac{\circ}{\varnothing}$ grams are obtained if the tube is left in while the oil is instilled. The pleurograms enable the sur- $\overrightarrow{0}$ geon to keep the mouth of the tube just within the 3 most dependent part of the cavity and also to watch the rate of re-expansion of the lung. The pleurograms should be repeated every two or three $\frac{0}{3}$ weeks. During the process of re-expansion the cavity may shrink concentrically towards its base, $\frac{\sigma}{3}$ in which case little or no alteration in the length and direction of the tube will be necessary; but $\frac{\rho}{3}$ the lung may move towards the chest wall in such a way that a loculus may become separated from the main cavity. In this case it will be necessary? to lengthen the tube so that the mouth enters the pocket so as to prevent it being nipped off from the $N$ main cavity (Fig. 6). Some complex empyema N cavities may require two tubes and it may be N necessary to use two or more separate entry sinuses, but the use of multiple rib resections delays the return of full function. If a long intrapleural tube has to be used the lung will eventually re-expand so that the tube is closely invested and ${ }_{0}^{+}$ the cavity is converted to a tube track. When this occurs the tube is maintained $\frac{3}{4}$ to $I$ in. shorter $\vec{\Phi}$ than the track. At the fortnightly pleurogram it $\frac{?}{\Phi}$ 

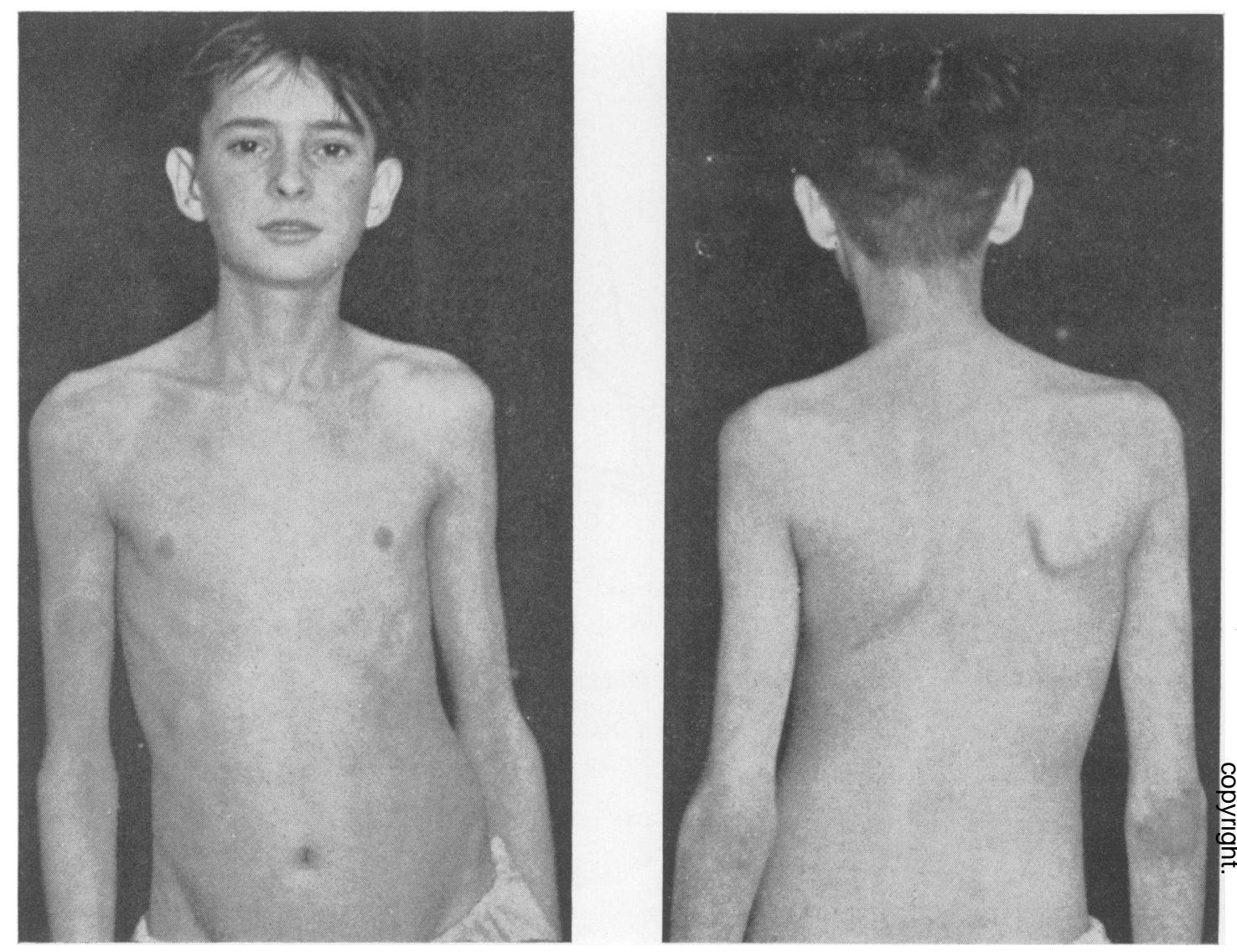

Fig. 8.-(a) The deformity of chronic empyema.

will usually be found that the end of the track has closed by this amount and the tube and the track are the same length. The tube is therefore shortened by another similar amount until finally, when the tube passes no more than $\frac{1}{2}$ in. into the pleural cavity, it can be removed. 'The total length of the tube just before it is discarded is usually about 2 in. in the normal but more in the obese patient.

The operation of decortication requires a full thoracotomy, the usual practice being to resect the greater part of the sixth rib from the tip of the transverse process to the anterior axillary line. The pleural cavity is then entered and the chest widely opened by a mechanical rib spreader. Pus, fibrin and debris are sucked out. The anaesthetist maintains the lung as fully expanded as possible and an incision is made, at a convenient point, through the fibrin layer, until the blue glistening pleural surface is seen. The fibrin layer can then be peeled away from the pleural surface by blunt and occasional sharp dissection. The entire visceral pleura must be freed and as this is done $\frac{3}{3}$ the lung progressively expands until it fills the chest (Fig. 7). It is difficult, if not impossible, to 3 . decorticate the parietal pleura, and it is not necessary to do so. Difficulty in decorticating the visceral pleura usually arises around the fringe of 0 the lower lobe where the fibrin layer passes? smoothly on to the diaphragm, and in clearing the diaphragmatic surface of the lower lobe. The mediastinal aspect of the lung is very seldom $N$ densely adherent since it rarely forms part of the $N$ wall of an empyema cavity. Although the object N is to separate the fibrin layer from the visceral $\sigma$ pleura this can seldom be achieved without tearing $\underset{\bullet}{<}$ the pleura. Multiple small alveolar fistulae are pro- $\frac{C}{0}$ duced, through which frothy blood is blown. Be- @ yond causing inconvenience to the operator they 0 are of no importance, and close spontaneously. The $\vec{\circ}$ lung being fully expanded, the chest is closed with $\stackrel{\mathbb{Q}}{\circ}$ two or three closed intercostal drains connected to $\frac{\vec{\nabla}}{\mathbb{0}}$ 
light suction pumps or water seals. It is essential to maintain the lung fully expanded until the pleural layers become adherent. There will be some oozing of blood from the operative area together with a reactionary effusion during the first few post-operative days, and if this and all the residual air in the pleural cavity is not removed the lung will fall away from the chest wall, which it reached at the end of the operation, and the empyema will re-form. To avoid this it is customary to use a tube at the apex of the chest, through the second or third intercostal space, and a second at the base, posteriorly. A third may be placed in the axilla. These tubes are removed as and when they cease to drain fluid or air, which occurs when the lung in re-expanding seals them off. Drainage can usually be discontinued after four or five days. The post-operative period requires very close supervision and frequent radiographic control.

A development of the operation of decortication, applicable to small localized empyemata, consists of the excision of the empyema cavity intact with its contents. As before, a long piece of rib is resected, overlying the centre of the empyema. The parietal pleura is not opened, however, but is stripped away from the chest wall by blunt dissection until the edges of the hard empyema wall are felt to give way to the normal soft pleura. The parietal pleura is incised around the edge of the empyema and the dissection continued along the deep surface of the empyema, in the plane between the visceral pleura and the fibrin layer. When this is completed the empyema can be removed like a cyst. The remainder of the pleural space is freed of light adhesions and the chest closed with drainage as described above.

\section{Chronic Empyema}

It remains to consider the causes and treatment of chronic empyema. By far the commonest causes are delayed or inadequate drainage or a combination of the two. Re-expansion of the lung is prevented by the thick fibrin layer deposited on the visceral pleura; movement of the chest wall, essential if the lung is to expand, is prevented by the similar deposit on the parietal pleura. The ribs fall together and eventually overlap, assuming a triangular shape on cross section. The intercostal muscles atrophy. A scoliosis develops with the concavity towards the side of the empyema. The shoulder on the affected side drops, the neck inclines to the empyema side and the head is carried vertically but above the middle of the clavicle (Fig. 8). Prolonged physiotherapy can do much to reverse these deformities provided that the empyema is properly drained. Other causes of chronicity are the presence of a foreign body in the empyema cavity-a piece of drainage tube, etc., but this is not commonly found. The investigation of the cause of chronicity in a well-drained empyema is more fruitfully directed towards the state of the bronchus and the lung. Bronchial obstruction, due to neoplasm, foreign body or stricture must be excluded by bronchoscopy, and if this shows no lesions then a bronchogram may show bronchiectasis.

The treatment of chronic empyema-provided the cause of the chronicity can be removedconsists firstly in a prolonged course of physiotherapy to obtain as much re-expansion and to reduce as much of the deformity as possible. Provided that the empyema is not so longstanding that fibrosis of the lung has occurred, decortication may be successful. All attempts to re-expand the lung having failed, it becomes necessary to obliterate the space by some plastic operation on the chest wall. A small empyema cavity may be obliterated by a muscle graft operation, making use of the latissimus dorsi and the intercostal muscles to fill the space. The blood and nerve supply of the muscles used must be preserved, and the muscle flaps must be carefully designed for this purpose. Large chronic cavities can often be closed by a postero-lateral thoracoplasty, long segments of ribs being removed. It is always necessary to remove the upper ribs although they may lie above the cavity. If they are left, the chiest wall will not fall in adequately. The extent of the thoracoplasty towards the base depends on the site of the lowest point reached by the empyema cavity; one or two ribs below this level must be removed. The operation, consisting as it does of the total or subtotal resection of nine or ten ribs, is carried out in two or three stages and is one of considerable difficulty, because the ribs are triangular in shape, overlapping and brittle. In addition, there is profuse haemorrhage from the chronically inflamed periosteum and chest wall tissues generally. Roberts' operation consists essentially of raising the parietal wall of the empyema as a flap, which is mobilized by removing from it the ribs and scar tissue. It is then allowed to fall in and adhere to the visceral wall. A considerable raw area is left to heal by granulation.

\section{Tuberculous empyema}

The treatment of this condition is so closely bound up with the treatment of the tuberculous disease in the underlying lung, that it cannot be considered separately. Since most tuberculous empyema arise in the course of artificial pneumothorax treatment, the empyema can usually be controlled by repeated aspiration, and re-expansion of the lung. The immobilization of the lung provided by the thickened pleura and somewhat 
sunken hemithorax may provide some insurance against re-activation of pulmonary disease. Should the state of the lung be such that re-expansion is undesirable or impossible, thoracoplasty will both obliterate the empyema and allow the lung disease to heal. If there is gross destruction of the lung, with an associated empyema, extras pleural pneumonectomy, excising both the lun and the empyema, by dissection in the extra-. pleural plane, may be indicated.

BIBLIOGRAPHY

BARRETT, N. R. (1950), 'Techniques in British Surgery.' Philadelphia. W. B. Saunders.

\title{
ON THE TEACHING OF GERIATRICS
}

\author{
By T. N. RudD, M.D.(London), M.R.C.P. \\ Physician, the Belmont Hospital, Tiverton, Devon
}

Physicians who practise in hospitals devoted to the care of the elderly will be in general agreement with the view that many highly experienced and otherwise excellent doctors are nevertheless lacking in their approach to disease in old age. They adopt what will later be described as a 'negative approach,' characterized by an attitude of frustration and laisser-faire. When the doctor concerned is a general practitioner his attitude will, in addition, be coloured by the great difficulties which attend the care of the very old in their homes. There is, however, another and a positive approach to the subject, the adoption of which has resulted in many improvements, made during the last decade, in the care of the elderly in hospitals. More intensive study of old people's illnesses has led to the development of a technique of geriatrics. Knowledge of this technique is at present in its early childhood and so far there has been, in this country at least, little organized teaching of the subject. Textbooks on the diseases of the aged have appeared in America, where there is also a bimonthly journal (Geriatrics) ' devoted to research and clinical study of the aged and ageing.' In this country no textbook has been produced, while such articles as have appeared under geriatric titles have been usually of an administrative rather than a clinical nature. Clinical problems of disease in the elderly have received little attention from British teaching centres, although at least one teaching hospital in London has appointed a lecturer in geriatrics. The urgency of the problems of disease in the aged warrants a much more intensive teaching of the subject.

When teaching of such a nature is considered, several great questions immediately present themselves. These can be summarized under the headings Why? When? Whom? Where? What and How? It is under these headings that the teaching of geriatrics will be considered.

\section{Why Should Geriatrics be Taught?}

Geriatrics should be taught for three main reasons:-

(I) There is considerable ignorance among sections of the medical and nursing professionstas to what can be achieved by a positive, active approac $h$ to therapy, even in the very old. The variations $w h i h$ diseases show, when they presento themselves in aged subjects, are not, as yet, widelp understood and many disease conditions remai $\vec{B}$ untreated, either because cure at such an age is considered not worth while, or because the risk of therapy has not been balanced against the dis? advantages of leaving the patient untreated. Mucto more treatment, both medical and surgical, could advantageously be given in the later years of life The completion of second stages of prostatectom operations is a case in point.

(2) It is economically imperative to keep the elderly self-supporting physically, and indepens dent of care by relatives and hospitals. Sheldop (1950) has pointed out that it is probable that the. wage-earning population is already bearing itto maximum load of chronic sickness and that it cannot carry any additional burden withou reducing industrial output. Unless the elderly are less dependent in the future (when their numbers will be proportionately greater than today) that they are at present, the country will be faced wit a grave problem.

(3) Preventive medicine in recent decades ha\$ added years to the life of the community. Thes years will be neither happy nor fruitful unles? 